OPEN ACCESS

Edited by:

James Martin Boyle, University of Strathclyde,

United Kingdom

Reviewed by:

Sue Pearson,

University of Leeds, United Kingdom

Jean Ware,

Bangor University, United Kingdom

*Correspondence:

Olympia Palikara

olympia.palikara@roehampton.ac.uk

Specialty section:

This article was submitted to Special Educational Needs,

a section of the journal

Frontiers in Education

Received: 18 November 2017

Accepted: 03 April 2018

Published: 19 April 2018

Citation:

Palikara O, Castro S, Gaona C and Eirinaki V (2018) Capturing the Voices of Children in the Education Health and Care Plans: Are We There Yet?

Front. Educ. 3:24

doi: $10.3389 /$ feduc.2018.00024

\section{Capturing the Voices of Children in the Education Health and Care Plans: Are We There Yet?}

\author{
Olympia Palikara*, Susana Castro, Carolina Gaona and Vasiliki Eirinaki \\ School of Education, University of Roehampton, London, United Kingdom
}

The need for a focus on the voice of children with special educational needs and disabilities (SEND) has received increased recognition internationally both in policy and research. In England, this was emphasized in the new special educational needs framework introduced in 2014. As part of this new policy, children with disabilities and/or additional needs can receive an Education Health and Care (EHC) plan. The EHC plan is a single document that should describe the children's strengths and needs in a multidisciplinary and holistic way. Section A of the EHC plan must include the child's own perspective. In this context there is much need for evidence on the quality of these new plans and in particular on the quality of the depictions of children's voices. The aim of this study was to address this knowledge gap by analyzing the depictions of children's voices and the process by which these were gathered in $184 \mathrm{EHC}$ plans of children with SEND attending mainstream and special schools in the Greater London area. The content analysis of the section concerning the children's voices was conducted using the categories of a multi-dimensional classification system, which includes aspects relating to the child herself, but also to her environment and relationships - the International Classification of Functioning, Disability and Health (ICF). The findings revealed high levels of variability in the way the voices of children were captured, including the methods used to ascertain their views. Additionally, the type of school that the child was attending seemed to play a significant role on how his/her voice was captured, favoring mainstream schools. The findings of the present study provide the first set of evidence-based data concerning the quality of the content of the newly introduced EHC plans and are discussed in light of the implications they have for policy, practice and further research in the area.

Keywords: special educational needs (SEND) code of practice, voices, children, international classification of functioning, disability and health (ICF), education, health and care (EHC) plans

\section{INTRODUCTION}

Capturing the voices of children with special educational needs and disabilities (SEND) has increasingly been recognized as a key element of relevant policy initiatives internationally (United Nations, 2006; Porter, 2014). In England, there is increased emphasis on participation of children and young people in the decision-making process through the new SEND Code of Practice, which was introduced following the enactment of the Children and Families Act (CFA) 2014 (Department for Education and Department of Health, 2015). These reforms have been described as the most 
substantial ones in the UK over the last two decades (Department for Education, 2014; Norwich and Eaton, 2015; Curran et al., 2017). The CFA introduced the Education, Health and Care plans (EHC plans) to replace previous statements of Special Educational Needs. The EHC plan is a single document that should describe the children's strengths and needs, in a multidisciplinary and holistic way and portraying the child's own perspective. Although capturing the views of children is a key element of the new SEND policy, there is limited systematic evidence to date on how the voices of the children are depicted in the EHC plans and on the methods used to ascertain their voices. To the authors' knowledge, this is the first study which aims to address this gap by systematically analyzing the EHC plans of children attending mainstream and special schools in order to examine how their voices are being captured.

\section{The New Special Educational Needs Framework in England}

Some of the changes introduced by the new SEND framework concerned the definition of SEND; the emphasis on multi-agency team work in achieving an integrated, holistic assessment of the child or young person; the extension of the age range for the provision of SEND from birth to 25 years of age; and the childcentered approach, in which eliciting the voices of the children and young people themselves was considered a key element of their assessment and intervention. Additionally, as part of this SEND policy framework, Education Health and Care (EHC) plans replaced the Statements of SEN.

The Code of Practice provides an outline of the elements that each section of the EHC plan should contain (Department for Education and Department of Health, 2015). Section A of the EHC plan must include the child's own voice. The EHC plan is drawn up by the local authority (LA) following an education, health and care assessment which has concluded that an EHC plan is necessary in order for the child's needs to be met and also following consultations with relevant teams of professionals, parents and the children themselves. Therefore, the new SEND policy highlights the principle of child participation in decisionmaking. Additionally, it points out the legal duty of LAs to enable children and young people through the development of appropriate processes to express views, to be consulted and to take an active part in decision-making on ways of achieving the best possible outcomes.

\section{The Voices of Children With SEND}

The notion that the voices of children and young people with SEND should be heard and that they should be actively involved in decision making about their education, health and social care is not a new one and has been described and pointed out before in policy and legislation documents both in the UK and internationally (Children Act, 1989; Gray et al., 2006; United Nations, 2006; Palikara et al., 2009). Policy changes have taken place with the voice of the child in the center (Children Act, 1989; Individuals with Disabilities Education Act, 2004), but there still appears to be a gap between policy and practice (Porter, 2014). Although the values and ideologies of these policy documents are important, the empirical evidence on the effectiveness of these legislation documents in capturing the voices of children is still rather scarce. For example, in England, there is no systematic evidence on the quality of the methods by which the voices of children and young people were captured in previous legal documents describing their needs-the Statements of SEN-although critiques on Statements do exist (Warnock and Norwich, 2010). The evidence concerning the effectiveness of the EHC plans in capturing the voices of children since the reformed system was introduced are even narrower. In 2017, the Department for Education published the findings of a commissioned study examining the views of parents and children on EHC plans (Adams et al., 2017). It was reported that two thirds of the children that took part in the survey were satisfied with the process followed for obtaining their EHC plan and that this would help to achieve the outcomes agreed on the actual plan for the child or the young person. Interestingly, it was reported that only $44 \%$ of the children and young people were being directly asked if they wanted to take part and it was much less common for them to be given choices of how to take part (19\%) in the Education, Health, Care assessment process. It was also noted that the chances of children/young people being actively involved in the process seemed related to the child's age and whether they previously had a Statement of SEND. Along the same lines, two other recent reports (National Autistic Society, 2016; Scott, 2016) described the level of satisfaction of parents and children since the introduction of the reformed system but neither provided evidence deriving directly from the children's EHC plans documents.

The process or methods for eliciting the views of children and young people do not come without particular challenges. Previous research has highlighted that capturing the voices of children and young people is not unproblematic and requires use of appropriate methodology, meticulous planning and care in eliciting and interpreting valid responses (Whitehurst, 2007; Palikara et al., 2009). Whitehurst (2007) argued that this is particularly challenging when capturing the views of children with profound and complex needs and called for the need to develop appropriate methodologies and strategies to meaningfully engage in such processes with the children themselves. Palikara et al. (2009) examined the views of adolescents with a history of Specific Language Impairment during the transition from secondary education to post-16 and argued that the young people were aware of their history of SEND and were able to offer valid accounts of the effect that these difficulties had on different aspects of their lives including education. The findings of this study also highlighted important methodological issues in eliciting the views of children with SEND including the value of professional experience and the use of "narrative coherence" (Hauser et al., 2006), which concerns the integration of personal experience and circumstances in a meaningful story, as a useful methodological tool in ascertaining the views of these children. Although the above studies provided some valuable evidence concerning the challenges and the opportunities related to eliciting the views of children with SEND, it should be noted that none of them has examined ways by which the voices of these children are actually being depicted in official legal documents, such as their Statements 
of SEN, or EHC plans, which usually accompany the children and young people throughout their whole educational path and play a significant role in determining outcomes, aspirations expectations and provision among other things.

In order to address some of the gaps identified in the literature above, we gathered some novel data by investigating the quality of the newly introduced EHC plans specifically regarding the nature of the content included in section A-the voice of the child. In particular, we aimed to address the following four research questions:

1. Have the children's voices as presented in the section A of the EHC plans been obtained directly from the child?

2. What methods were used in order to elicit the views of the children on the EHC plan?

3. In section A of children's EHC plans was the focus on abilities, disabilities or both?

4. Were there any differences in the depictions of children's voices in EHC plans from mainstream schools when compared to those from special schools?

In order to address the research questions above, the International Classification of Functioning, Disability, and Health for Children and Youth (ICF-CY), was used as a theoretical framework and coding system. In 2007, the WHO published the ICF-CY, which followed the publication of its adults' version (ICF) in 2001. The ICF, which is considered to provide a third viewpoint, beyond the medical model and the social model of disability in the conceptualization of SEND, acknowledges that the source of the disability is the complexity of individual and social factors interacting to result in a unique individual functioning profile (Simeonsson, 2006). This ICF was selected as a theoretical and analytic framework for the purposes of the specific study because the multidimensional conceptualization of disability as described in the ICF-CY is closely aligned with the definition of SEND in the new SEND policy. Although the ICF-CY is not mentioned directly in the new policy, a rationale concerning the possible links between the two has been presented elsewhere (Castro and Palikara, 2016). Castro and Palikara (2016) have also argued that the body functions and the activities and participation codes introduced by the ICF-CY could be used to document the children's SEND in the EHC plans. Similarly, the environmental factors' codes could be applied to describe the environmental influence of contextual features on the child's participation, using a common language. The approach to disability as briefly described above was key for the adoption of the ICY-CY as the taxonomic system to serve the purposes of the present study.

\section{MATERIALS AND METHODS}

\section{Recruitment and Data Collection}

In order to proceed with the analysis of the EHC plans of children with SEND, a formal invitation letter was sent to a number of LAs in close proximity with the University of Roehampton. Once the support of the LA was obtained, formal invitation letters with informed consent forms for head teachers, Special Education Needs Coordinators (SENCOs) and parents were sent to all schools within the LA that had expressed their interest in participating in the study. Meetings were held with the representatives of the schools that expressed interest in the study, in order to explain the scope, process and ethical standards of the project and how the results would be reported. Additionally, meetings were held with parental associations in some LAs, where the project was presented, and parents invited to participate. For each school that agreed to participate, the key contact person liaised with the parents to obtain opt-in consent from them to release the EHC plan of their child/children. Both online and physical informed consent forms were issued and accepted; the content of the online form was strictly the same as the physical form. When parents expressed their interest in participating directly to the team, signed informed consent forms were sent to us without school mediation. As an incentive to participate in the study the team made the commitment of feeding back the general results of the study to both parents and professionals. Once the informed consent forms were received by the team in either online or physical format, the team would contact the parent/key professional for the anonymized EHC plan to be sent to us in password-protected format.

\section{Participants}

The EHC plans included in the analysis describe the education health and care needs of 52 girls and 132 boys in 92 mainstream and 92 special schools, across 9 London LAs. 184 EHC plans were analyzed from a larger dataset of EHC plans that have been received by the research team. These 184 plans were selected on the basis that they were the ones fully completed at the time of data analysis, and included a final version of all sections of the EHC plan. The date of birth was made available for 175 of the 184 children; mean age was 11.21 years, with a standard deviation of 4.04; the youngest participant was 3 years old and the oldest participant 21 years old.

\section{Ethical Issues}

The study was approved by the Ethics Research Committee of the University of Roehampton, which adheres to the British Psychological Society guidelines. Written and informed consent was obtained from all adult participants as well as from the parents/legal guardians of all non-adult participants. The plans included in the analysis were anonymized. Confidential information regarding the name of the LA, parents' or schools' contact details was only accessed by the core members of the research team. This data was permanently kept in passwordprotected files on the university computers (when in online format) and in locked cabinets in the team's research office.

\section{Data Analysis}

The content of section A of the plans included in this study was analyzed through a process of systematic content analysis. Two types of content analysis were included sequentially. First, an inductive process of content analysis was adopted to ascertain whether general themes would emerge from the reading of section A of the plans. As a result of this analysis, it was noted that this section contained information on abilities/strengths of the child and on disabilities/functioning issues of the child; similarly, 
some plans use the first person ("I") while others refer to the child using the third person. However, in many of the plans using the first person, there were indications of the fact that maybe the child herself did not provide the information conveyed, as it was often characterized by a rather complex discourse in cases where children were reported not to have the functional abilities to express their view in such complex way. Therefore, as a first step and as a result of an inductive content analysis, frequencies were run for all plans regarding the following categories: number of plans that use the first person in section A; number of plans that report the process by which the voice/perspective of the child was obtained; number of plans with section A focusing on abilities, disabilities or both.

The second and following type of content analysis adopted was a deductive content analysis using the International Classification of Functioning, Disability and Health for Children and Youth (WHO., 2007; ICF-CY, 2007). This is a classification system regarded by the $\mathrm{WHO}$ as the gold standard for classifying disability in children and it has been extensively used in other studied to support the exploration of disability-related content (e.g., Castro et al., 2014; Klang et al., 2016; Osborne et al., 2017). Using the ICF-CY categories as pre-defined codes for deductive content analysis supports a deeper examination of the nature of the content included in section A of the plans. This second phase of analysis followed specific steps: (1) identification of units of meaning within the text relating to either abilities/strengths of the child and those referring to disabilities/functioning issues and also those referring to environmental issues; meaning units are hereby defined as the constellation of words or statements that relate to the same central meaning (Graneheim and Lundman, 2004); (2) all meaning units in all three categories (abilities, functioning issues, and environmental issues) were coded using the ICF-CY system and following coding procedures used in previous studies, namely the ICF linking rules (e.g., Cieza and Stucki, 2005; Castro et al., 2014; Cieza et al., 2016); the linking rules were developed for the specific purpose of linking content with the ICF, and include specific notations for each type of content that cannot be linked to the classification and guidelines on how to choose the ICF code to be linked to the meaning unit under analysis; (3) all meaning units were firstly coded by an experienced researcher with training on the ICF-CY system; (4) $5 \%$ of all meaningful units regarded as "abilities" and 5\% of all meaning units regarded as "disabilities" were double-coded by a second independent researcher also trained on the ICFCY to ensure reliability of criteria when coding. The choice of units to be double-coded was made through random selection of numbers on a computerized web-application; each number randomly selected would correspond to one EHC plan and the first 2 meaning units of that plan would be double-coded until $5 \%$ of the total was reached. Frequencies of meaning units were computed per ICF-CY component, abilities, disabilities and environmental issues and per category within these components. Demographic variables regarding date of birth, gender, age, LA and type of school (special or mainstream) were also recorded and included in the analysis.

We also tested whether there were differences between LAs and types of school in regard to: the use of the first person
TABLE 1 | Frequency of plans per local authority.

\begin{tabular}{lc}
\hline Local authority & Number of EHC plans obtained \\
\hline 1 & 66 \\
2 & 36 \\
3 & 27 \\
4 & 37 \\
5 & 3 \\
6 & 8 \\
7 & 4 \\
8 & 1 \\
9 & 2 \\
Total & 184 \\
\hline
\end{tabular}

in the section $\mathrm{A}$, the focus on abilities or disabilities and also regarding the content of the plans as coded by the ICF-CY system. These analyses were performed using non-parametric tests with R studio.

\section{RESULTS}

A total of 184 plans were included in the analysis. Twenty-six plans that were received with parental consent were not included in the analysis because the content of section A (the child's perspective) was missing-the section was either not completed or the content was extremely limited. The plans included in the analysis describe the education health and care needs of 52 girls and 132 boys in 92 mainstream and 92 special schools, across 9 London LAs. The date of birth was made available for 175 of the 184 children; mean age is 11.21 years, with a standard deviation of 4.04; the oldest young person is 21 years old and the youngest is 3 years old. Table 1 describes the number of EHC plans included in the analysis per LA. Because some LAs provided very few plans, only the first four were included in the inferential analysis.

The LAs included in the inferential analysis differ substantially in terms of their demographics. According to the 2015 Indices of Deprivation, LAs 2 and 4 are within the 10\% least deprived in the country, while LAs 3 and 1 are in the 50\% more deprived. More details about the socio-economic characteristics of these boroughs cannot be included for preservation of confidently and anonymity.

\section{Results of the Induction Analysis}

As a result of the inductive content analysis performed with the $184 \mathrm{EHC}$ plans included in this study, it was observed that 117 plans (63.6\%) used the first person in section A of the plans. However, amongst these, many show some indication that the voice of the child was not directly obtained, as the firstperson discourse seems rather complex for the pattern of needs described in the child's profile. For example, "I enjoy interacting with people including strangers" (participant 57, characterized in the plan as having a global developmental delay, performing way below age related expectations and needing visual cues to communicate at 6 years of age) and "I can give an answer from 2 or 3 choices, point to what I want or show what I want" (participant 


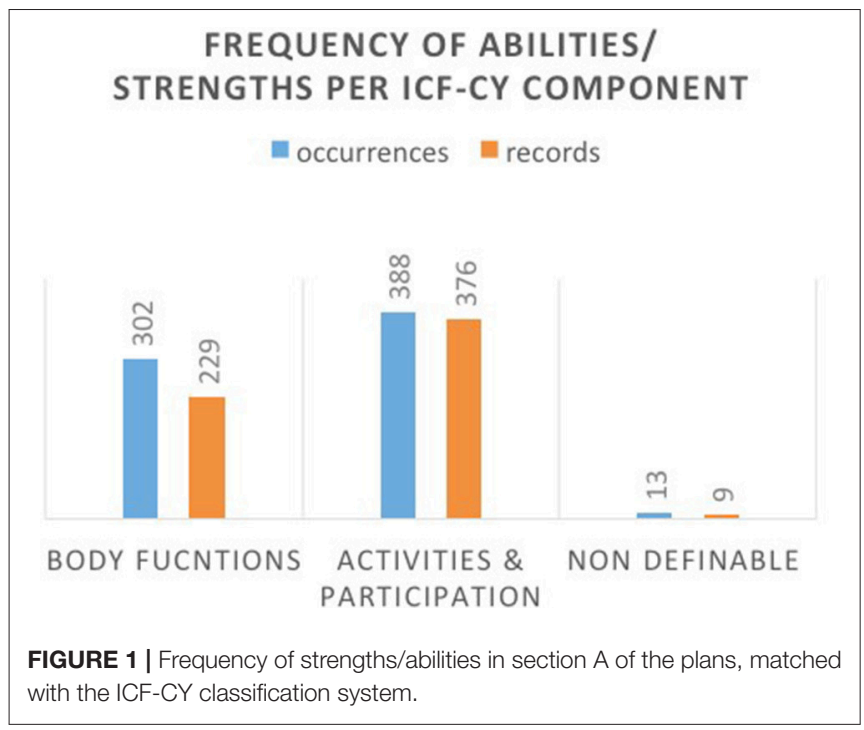

56 , who is described in the needs section of his plan as "not engaging without support" and just "beginning to understand routines and expectations in school at 6 years of age"). Therefore, it is difficult to ascertain whether the use of the first person in the great majority of the plans actually represents the real number of children there were indeed heard. Only 31 plans of the 184 (16.8\%) report the methods by which the voice of the child was obtained. Often, there is mention to the fact that the parent filled in the section of the plan in representation of the child, but there is no indication in the great majority of these plans on how the child's views are understood by the parent or other representing adults. When looking at whether the plans focus on abilities, disabilities or both in section $\mathrm{A}$, it was observed that the great majority of plans focus on both (91.3\%); For example, participant 197 "turns the pages by himself" but "doesn't like others being in his personal space and can become upset when this happens.”

\section{Results of the Deductive Analysis}

From the deductive content analysis performed using the ICF$\mathrm{CY}$ categories as a pre-defined classification of domains to link with the content of section A of the plans, the following was observed: 709 meaning units were identified as relating to abilities or strengths of the children and 1,010 meaning units were identified as relating to disabilities or functioning issues of the children; therefore, even though the great majority of plans focus on both abilities and disabilities as described above, there a majority of content units focusing on functioning issues rather than strengths. Additionally, 277 meaning units were identified as relating to environmental issues.

Figure 1 shows the frequency of plans and the total number of occurrences of each meaning unit, within the ones classified as abilities/strengths of the child. The figure shows that most abilities identified in section A of the EHC plans refer to Activities and Participation aspects, followed by Body Functions; some aspects were classified as non-definable for being rather vague and consequently not susceptible of being classified with a
TABLE 2 | Most common ICF-CY codes used to describe abilities/strengths.

\begin{tabular}{lcc}
\hline ICF-CY code & Occurrences & Records \\
\hline Motivation functions & 191 & 141 \\
Temperament and personality functions & 81 & 58 \\
Interactions with peers & 39 & 38 \\
Communicating with gestures & 24 & 20 \\
Speaking & 20 & 19 \\
Eating & 18 & 18 \\
Calculating & 14 & 14 \\
Non-definable & 13 & 9
\end{tabular}



FIGURE 2 | Frequency of functioning issues in section A of the plans, matched with the ICF-CY classification system.

specific system such as the ICF (for example, participant 33 was described as having said that she needs help with "gross motor skills" in section A). Table 2 discriminates the specific aspects of functioning that were most commonly mentioned as abilities/strengths of the child: motivation functions appear 191 times (occurrences) in 141 plans (records). It is very common that the child or representative describes what the child enjoys doing. These were all classified as motivational aspects. The second most common aspect identified as abilities of the children was temperament and personality functions (children being described as "loving," "sociable," etc.; these appeared 81 times in 58 plans Other aspects identified as abilities were observed in a minority of plans, including interactions with peers, communicating through gestures, speaking, eating and calculating.

Figure 2 shows the frequency of plans and the total number of occurrences of each meaning unit, within the ones classified as disabilities/functioning issues of the child. Once again, Activities and Participation are the most common type of functioning issue observed, followed by Body Functions. There are only 8 occurrences corresponding to 8 records of Body Structures identified as functioning issues; 55 occurrences in 45 records were non-definable.

Table 3 shows the specific type of content assigned to functioning issues. The most common functioning issue observed was emotional functions (often linked to emotional regulation issues, such as aggressive outbursts or tantrum 
TABLE 3 | Most common ICF-CY codes used to describe functioning issues.

\begin{tabular}{lcc}
\hline ICF-CY code & Occurrences & Records \\
\hline Emotional functions & 82 & 62 \\
Speaking & 60 & 59 \\
Non-definable & 55 & 45 \\
Accepting novelty & 38 & 32 \\
Eating & 37 & 35 \\
Dressing/undressing & 34 & 34 \\
Regulation of emotions & 31 & 25 \\
Toileting & 31 & 31 \\
Sensory functions & 29 & 28 \\
Writing & 27 & 27 \\
Directing attention & 26 & 26 \\
Reading & 24 & 24
\end{tabular}

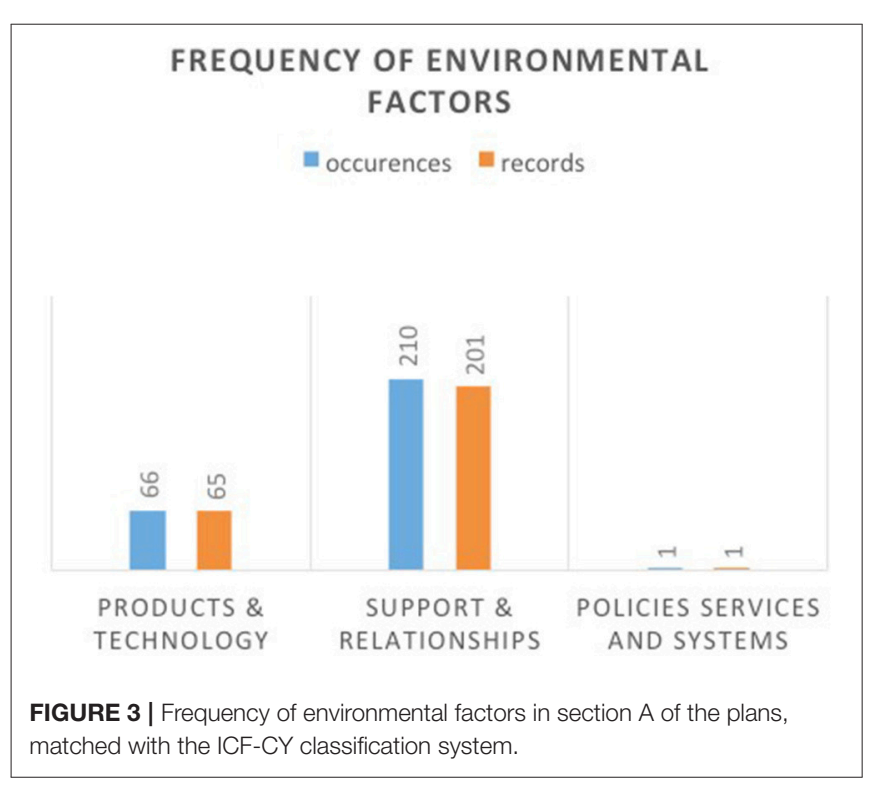

behaviors). Speaking is the second most common functioning issue. Non-definable issues due to their vagueness and lack of clarity were the third most commonly identified.

Figure 3 shows the frequency of plans and the total number of occurrences of each meaning unit, within the ones classified as environmental factors relevant for the child. The majority of meaning units link to support and relationships, followed by products and technology. The specific environmental aspects most commonly included in section A were the support of the immediate family, which was mentioned in the great majority of plans (151); products and technology include medication and communication aids.

\section{Results of the Inferential Analysis}

Series of non-parametric tests (Mann-Whitney and KruskalWallis) were run to test differences between LAs and type of school regarding the inclusion of specific abilities, disabilities and environmental issues in section A of the EHC plans. Nonparametric tests were used given the categorical nature of the variables under analyses. In fact, the Kruskall-Wallis test $(\mathrm{H})$ "is approximately chi-square distributed, meaning that the probability of getting a particular value of $H$ by chance, if the null hypothesis is true, is the P value corresponding to a chi-square equal to $H^{\prime \prime}$ (McDonald, 2014, p. 157). No differences were found between LAs or type of school in this respect.

Similar analysis was run to test the difference between LAs and type of school in relation to the use of the first person in section A of the plans, the quality of the report on how the voice of the child was obtained and regarding a stronger focus on abilities, disabilities or both. It was found that LA 1 uses the first person significantly less than LA 2 and $4[H(8)=39.41, p \leq 0.05]$. Local authority 3 describes the method for obtaining the child's voice significantly more than the other LAs $[H(8)=72.31, p \leq 0.05]$. Mainstream schools report the method for obtaining the voice of the child significantly more often than special schools $(U=11.81$, $p \leq 0.001)$.

\section{DISCUSSION}

To our knowledge, this is the first study which aimed to provide systematic evidence concerning the quality of the newly introduced EHC plans, in relation to how the voices of children with SEND attending mainstream or special schools were captured. The content of $184 \mathrm{EHC}$ plans of children with SEND attending mainstream and special schools in the Greater London area were analyzed using the ICF as theoretical and analytical framework. The findings of the study showed great variability between LAs in the way that they were capturing the voices of the children and also in the methods employed to ascertain their views. Although most of the EHC plans included some information on children's abilities/strengths, the analysis of these units showed that they were rather limited in scope and revealed little about what the child was good at. Significant differences were found in the way the voices of the child were captured depending on the type of school attended, with mainstream schools providing more detail.

\section{Variability of EHC Plans}

The results of the study highlight the high level of variability observed in the way different LAs were capturing the children's voices. The data collected in this study was gathered between 2015 and the end of 2016 and therefore cannot represent the current national trend; this is still to be known upon completion of conversions of statements into EHC plans. However, the present study includes a variety of EHC plans, developed within LAs that differ substantially regarding their ways of operating and availability of resources. As mentioned in the sample description, the areas included in the main analysis differ in terms of their deprivation indices. This is interesting if we consider the observation that some of the quality aspects of the plan (section A) differed significantly between LAs; these differences might reflect different procedures in designing and implementing the EHC planning process, but also the resources (and often the lack of them) available to support these procedures. 
The differences found between LAs may also reflect the lack of national guidelines for the development of EHC plans, placing the accountability for the quality of the process within the LA only. Given that their resources differ substantially, the lack of such guidelines is directly affecting the quality of the EHC plans that will be made available for children and families in more deprived areas. Future research should endeavor to provide evidence to support the development of guidelines to improve the quality and comparability of the EHC planning process across the nation. Although Statements of SEN have been criticized in the past on how useful they were as tools in meeting the needs of children (Audit Commission, 2002; Norwich, 2005), the evidence base for the effectiveness and usefulness of EHC plans for children, young people, families and professionals remains to be seen. The findings of this study revealed that mainstream schools tend to report significantly more often the methods used in obtaining the child's voice when compared to special schools. This is an interesting result as one could deduct that special schools would master techniques of accessing the children's voices better than mainstream schools that often do not have the specialized staff. However, it may also be the case that special schools support children with more severe functioning issues than mainstream schools and therefore find the process of accessing their voices even more challenging. Indeed, previous research in the area of ascertaining the voices of children with complex needs has underscored the particular challenges that professionals may experience during this process (Whitehurst, 2007; Palikara et al., 2009). Additionally, it should be noted that the results of the current study may not be generalizable in terms of the differences found in the quality of the EHC plans between mainstream and special schools; this would not be achievable considering the currently observed delay in the completion of EHC plans. In fact, between January 2016 and January 2017, only $32.7 \%$ of the children and young people with statements had received a converted EHC plan (Department for Education, 2017), which is illustrative of the slow pace of the conversion process. Perhaps national guidelines for the development of EHC plans should also include a range of evidence-based methods and strategies to use with children of various functioning levels in order to gather their views. Future research should address the link between the quality of the EHC plans developed and the pattern of needs of the children in receipt of those plans.

\section{Description of Abilities and Disabilities in the EHC Plans}

The findings of the present study illustrated that even though the majority of EHC plans included some information on the abilities/strengths of the child, this seemed to be much more often related to what the child likes/enjoys doing, than to what the child is good at. Even though the information on motivational issues is regarded as of great importance, it might be equally relevant to include actual aspects of functioning that can be linked to day-to-day successes, things that the child is good at, to support educational interventions. These issues could integrate national-wide guidelines on how to develop high quality EHC plans. Similarly, temperament and personality functions are often mentioned as abilities/strengths of the child. This happens as in most cases there is a description of the child's general behavior (using terms such as "loving," "caring," "sociable" or "cute"), more than actual behavioral dimensions that can be prompted and measured as the basis for educational interventions and monitoring.

Regarding the specific types of disabilities identified in the analyzed plans, it is worth noting that the code non-definable was the third most frequent code linked to these meaning units. The reason for this was the rather vague nature of many of these statements. For example, "has issues around language development" or "needs to be more independent" are rather general statements that do not support potential interventions and do not illustrate the highly specialized work of a multidisciplinary team of experts that should have developed the plan bringing together their expertise. On the contrary, these are general, not specialized statements that could apply to many children with or without education, health and care needs. Non-definable statements identified in this study also refer to diagnostic data which is often presented as a way of describing a general need, but not necessarily informing educational intervention, or actions to take place in an educational setting (for example, "has epilepsy," but no information is available on the frequency and severity of seizures and functional consequences for the child's participation). The issues of quality observed in these plans suggest that there might be other factors influencing the quality levels of the EHC plans developed and these might be the training and specialization characteristics of those involved in EHC plan development. To date there are no national guidelines, standardized procedures or minimum requirements to be met by those who are involved in the process. The data observed in this study might be a reflection of such dearth of specialization.

\section{The Use of ICF As Theoretical and Analytical Framework}

In the present study, the use of the ICF was a valuable theoretical and analytical framework to enable the identification of quality issues with a greater level of specificity, looking at the actual nature of the content included in the plans. The biopsychosocial nature of this classification system split into Body Functions and Structures, Activities and Participation and Environmental Factors seems to be a good fit when trying to analyse the quality of documents that aim to be holistic in their descriptions of education, health and care aspects. This suggests that perhaps the use of such system as a supporting tool in the developing stage of the EHC plans could potentially help to improve their quality-by reducing vague statements, introducing standard levels of information of social and individual nature as well as by providing a common language between professionals from different areas of intervention. The ICF is of particular relevance for the English context as it has the potential to address key issues in the current SEND policy including: ensuring parent and child access rights to an adequate, relevant and user-friendly assessment; adopting an interactive causal model and holistic model of disability, which is relevant to a range of areas of 
functioning; covering personal strengths, not just difficulties; and supporting inter-professional collaboration (Castro and Palikara, 2016; Norwich, 2016).

\section{CONCLUSION}

This study examined how the voices of children with SEND attending mainstream and special schools were captured on their EHC plans. However, it should be noted that the pattern of needs that the child experiences was not taken into account in this analysis. This was a deliberate decision in order to allow for the emergence of themes that they may be universal concerning the quality of the EHC plans, regardless of the type of SEND. However, future studies should consider whether there are significant links between the children's patterns of needs and the quality of their EHC plans. Additionally, it would be useful to consider whether capturing the voices of children with profound and complex learning issues or rare developmental disorders, may affect the quality of how the voices of these

\section{REFERENCES}

Adams, L., Tindle, A., Basran, S., Dobie, S., Thomson, D., Robinson, D., et al. (2017). Experiences of Education, Health and Care plans: A Survey of Parents and Young People. London: Department for Education.

Audit Commission (2002). Competitive Procurement. London: Audit Commission.

Castro, S., and Palikara, O. (2016). Mind the gap: the new special educational needs and disability legislation in England. Front. Educ. 1:4 doi: 10.3389/feduc.2016.00004

Castro, S., Pinto, A., and Simeonsson, R. J. (2014). Content analysis of Portuguese individualized education programmes for young children with autism using the ICF-CY framework. Eur. Early Child. Educ. Res. J. 22, 91-104. doi: 10.1080/1350293X.2012.704303

Children Act 1989 (CA) (UK). Available online at: https://www.legislation.gov.uk/ ukpga/1989/41/contents

Cieza, A., Fayed, N., Bickenbach, J., and Prodinger, B. (2016). Refinements of the ICF linking rules to strengthen their potential for establishing comparability of health information. Disabil. Rehabil. doi: 10.3109/09638288.2016.1145258. [Epub ahead of print].

Cieza, A., and Stucki, G. (2005). Content comparison of health-related quality of life (HRQOL) instruments based on the international classification of functioning, disability and health (ICF). Qual. Life Res. 14, 1225-1237. doi: $10.1007 /$ s11136-007-9174-8

Curran, H., Mortimore, T., and Riddell, R. (2017). Special educational needs and disabilities reforms 2014: SENCos' perspectives of the first six months. Br. J. Spec. Educ. 44, 46-64. doi: 10.1111/1467-8578.12159

Department for Education (2014). Children and Families Act. London: Department for Education.

Department for Education and Department of Health (2015). Special Educational Needs and Disability Code of Practice: 0 to 25 years. London: HMSO.

Department for Education (2017). Special Educational Needs and Disability (SEND). Available online at: https://www.gov.uk/government/policies/ special-educational-needs-and-disability-send (Accessed 2 November 2017).

Graneheim, U. H., and Lundman, B. (2004). Qualitative content analysis in nursing research: concepts, procedures and measures to achieve trustworthiness. Nurse Educ. Today 24, 105-112. doi: 10.1016/j.nedt.2003. 10.001

Gray, P., Bullen, P., Duckett, L., Leyden, S., Pollard, I., and Skelton, R. (2006). National Auditof Support, Services and Provisions for Children with Low Incidence Needs. London: Department for Education and Skills. children are being captured, because of the difficulties inherent with eliciting the views of these children (Whitehurst, 2007). Furthermore, research aimed at examining the quality of how the voices of older young people with SEND are being captured, especially in preparation for adulthood is a noteworthy area for future research with important educational and clinical implications.

\section{AUTHOR CONTRIBUTIONS}

OP and SC: Have conceptualized and written the whole paper in collaboration, and the ideas provided are the result of shared reflection; CG and VE: Contributed to conducting the study and drafting and revisiting the intellectual content of the paper.

\section{FUNDING}

This project was funded by a British Academy/Leverhulme Small Research Grant (grant number SG142214).

Hauser, S. T., Golden, E., and Allen, J. P. (2006). Narrative in the study of resilience. Psychoanal. Study Child 61, 205-227. doi: 10.1080/00797308.2006. 11800771

Individuals with Disabilities Education Act 2004 (IDEA) (USA). Available online at: https://sites.ed.gov/idea/

Klang, N., Rowland, C., Fried-Oken, M., Steiner, S., Granlund, M., and Adolfsson, M. (2016). The content of goals in individual educational programs for students with complex communication needs. Augment. Altern. Commun. 32, 41-48. doi: 10.3109/07434618.2015.1134654

McDonald, J. H. (2014). Handbook of Biological Statistics, 3rd Edn. Baltimore, MD: Sparky House Publishing.

National Autistic Society (2016). School Report 2016: Two Years on, How is the New Special Educational Needs and Disability (SEND) System Meeting the Needs of Children and Young People on the Autism Spectrum in England? London: NAS.

Norwich, B. (2005). Inclusion: is it a matter of evidence about what works or about values and rights? Education 33, 51-56. doi: 10.1080/03004270585200091

Norwich, B. (2016). Conceptualising special educational needs using a biopsycho-social model in England: the prospects and challenges of using the International Classification of Functioning framework. Front. Educ. 1:5. doi: 10.3389/feduc.2016.00005

Norwich, B., and Eaton, A. (2015). The new special educational needs (SEN) legislation in England and implications for services for children and young people with social, emotional and behavioural difficulties Emotion. Behav. Difficult. 20, 117-132. doi: 10.1080/13632752.2014.9 89056

Osborne, C. L., Petersson, C., Graham, J. E., Meyer, W. J. III., Simeonsson, R. J., Suman, O. E., et al. (2017). The burn model systems outcome measures: a content analysis using the international classification of functioning, disability, and health. Disabil. Rehabil. 39, 2584-2593. doi: 10.1080/09638288.2016.12 39767

Palikara, O., Lindsay, G., and Dockrell, J. E. (2009). Voices of young people with a history of specific language impairment (SLI) in the first year of post-16 education. Int. J. Lang. Commun. Dis. 44, 56-78. doi: 10.1080/136828208019 49032

Porter, J. (2014). "Research and pupil voice," in The SAGE Handbook of Special Education, Vol. 2, 2nd Edn., ed L. Florian (Los Angeles, CA: SAGE Publications, Inc), 415-420.

Scott, L. (2016). SEND: The Schools and Colleges Experience: A Report to the Secretary of State for Education by Lee Scott: November 2016. London: Department for Education. 
Simeonsson, R. J. (2006). Defining and Classifying Disability in Children. Disability in America. Washington, DC: National Academic Press.

United Nations (2006). Convention on the Rights of Persons With Disabilities. Geneva: UN.

Warnock, M., and Norwich, B. (2010). Special Educational Needs: A New Look. London: Continuum International Publishing Group.

Whitehurst, T. (2007). Liberating silent voices-perspectives of children with profound and complex learning needs on inclusion. Br. J. Learn. Disabil. 35, 55-61. doi: 10.1111/j.1468-3156.2006.00405.x

WHO. (2007). International Classification of Functioning, Disability and Health for Children and Youth. Genève: WHO.
Conflict of Interest Statement: The authors declare that the research was conducted in the absence of any commercial or financial relationships that could be construed as a potential conflict of interest.

Copyright (๑) 2018 Palikara, Castro, Gaona and Eirinaki. This is an open-access article distributed under the terms of the Creative Commons Attribution License (CC $B Y)$. The use, distribution or reproduction in other forums is permitted, provided the original author(s) and the copyright owner are credited and that the original publication in this journal is cited, in accordance with accepted academic practice. No use, distribution or reproduction is permitted which does not comply with these terms. 